\title{
Measurement of Trans-Epithelial Electrical Resistance with EVOM2 and EndOhm Cup
}

\author{
Nicholas M. Mallek
}

University of North Carolina at Chapel Hill

Shaun D. McCullough ( $\nabla$ mccullough.shaun@epa.gov)

US Environmental Protection Agency https://orcid.org/0000-0001-6660-346X

\section{Method Article}

Keywords: TEER, epithelial, EndOhm, EVOM, trans-epithelial, barrier, pHBEC

Posted Date: November 3rd, 2021

DOl: https://doi.org/10.21203/rs.3.pex-1672/v1

License: (c) (i) This work is licensed under a Creative Commons Attribution 4.0 International License. Read Full License 


\section{Abstract}

Trans-epithelial Electrical Resistance (TEER) can be used as a measure of cell monolayer confluence, health, and integrity. An EndOhm chamber connected to an Epithelial Voltohmmeter (EVOM) may be used to take TEER measurements that are more reproducible than the chopsticks electrode. This method details how to take TEER readings using an EndOhm- 12 chamber and EVOM2 resistance reader. Read the manufacturer's manual carefully before adjusting any of the parameters below as the conditions described here were determined to meet the manufacturer's specifications. Volumes will need to be adjusted to accommodate substitution of the EndOhm-6 or EndOhm-24.

Disclaimer: The contents of this article have been reviewed by the US Environmental Protection Agency and approved for publication and do not necessarily represent Agency policy. Mention of trade names or commercial products does not constitute endorsement or recommendations for use.

\section{Introduction}

CAUTIONARY NOTES OR SPECIAL CONSIDERATIONS 1. The same growth medium must be used in both the apical and basolateral compartments during TEER measurement to avoid creating an ion gradient across the epithelial cell barrier. 2. Tissue culture incubator should have $\mathrm{CO} 2$ levels checked and recalibrated (if necessary) at least once per week. Incubators should also have external temperature monitoring probes. 3. Ensure the care of the EndOhm electrode and the calibration of the voltohmeter (below).

\section{Reagents}

\section{Equipment}

- EVOM2 Epithelial Voltohmeter (World Precision Instruments Inc, Sarasota, FL, USA)

- EndOhm-12 Chamber Cup (World Precision Instruments Inc, Sarasota, FL, USA)

· Dulbecco's Phosphate Buffered Saline (DPBS; ThermoFisher \#14190144)

- Growth medium appropriate for cells being used

- Humidified tissue culture incubator at $37^{\circ} \mathrm{C}$ with $5 \% \mathrm{CO}_{2}$ at ambient $\mathrm{O}_{2}$

- Biosafety cabinet (BSC)

- Cavicide (Metrex \#SKU13-1024)

- $70 \%$ Ethanol 


\section{Procedure}

NOTE: There is a methods document PDF version of this information included as a supplementary file.

\section{PROCEDURE}

\section{Preparation}

1. Warm cell culture media and DPBS in a $37^{\circ} \mathrm{C}$ water bath (hereafter referred to as "warmed" medium or DPBS).

2. Ensure that the EVOM2 unit is fully charged.

NOTE: If unsure, leave the EVOM2 unit plugged into an electrical outlet for 30 minutes before beginning your TEER assay.

NOTE: According to the manufacturer, taking TEER measurements while the EVOM2 unit is plugged into an electrical outlet can increase variability in your samples.

3. Acquire an additional 12-well plate ("holding plate") before starting any measurement.

a. A subset of your sample inserts will be held in this plate before transfer to the EndOhm-12 cup chamber.

4. Adjust the probe height on the EndOhm-12 unit (when applicable).

a. A manufacturer provided EndOhm-12 unit spacer can be used to reset the probe height on the EndOhm-12 cap probe to the factory standard.

b. Place a blank insert into the EndOhm-12 cup chamber, and the spacer inside the blank insert.

c. Loosen the locking nut on the cap probe and rotate the cap to change the height of the electrode until it touches the spacer.

d. For experimental applications, the cap probe electrode should be adjusted to be submerged in apical medium. 
5. Clean the EndOhm-12 by rinsing the cap and chamber with distilled water, then alcohol, then distilled water again and allow to dry before reassembling.

6. Clean the working surface of the biosafety cabinet (BSC) with Cavicide, then spray the working surface with $70 \%$ ethanol and wipe with paper towel(s).

a. Allow surface to air dry before putting materials into the BSC.

\section{Measuring blank resistance}

1. Place the EndOhm-12 cup chamber and the holding plate into a cleaned BSC.

2. Add $3.0 \mathrm{~mL}$ of warmed cell culture media to the EndOhm-12 cup chamber using a $5 \mathrm{~mL}$ serological pipet.

3. Place the blank insert into the holding plate and add $500 \mu \mathrm{L}$ of cell culture media to the apical compartment using a P1000 pipette.

4. Transfer the blank insert into the EndOhm-12 cup chamber.

a. Check for bubbles underneath the blank insert.

b. Remove the insert and transfer the insert back into the EndOhm-12 cup to remove any bubbles.

c. Placing the insert into the EndOhm-12 cup at an angle can reduce the frequency of bubbles.

5. Plug the EVOM2 connecting wires into the EndOhm-12 cup chamber, place the EndOhm-12 into a cell culture incubator, and close the door (Figure 1).

6. Switch the EVOM2 on. 
a. The blank resistance readings should stabilize within a few seconds.

7. Switch the EVOM2 off and return the EndOhm-12 to the BSC.

8. Transfer the blank insert back to the holding plate.

\section{Measuring sample resistance}

1. Remove samples from the cell culture incubator and transfer to a BSC.

2. Transfer your first three Transwell inserts to be assayed to the holding plate.

a. Transferring three samples to the holding plate at a time allows the remaining samples to be returned to the climate-controlled environment of the cell culture incubator.

3. If medium is present in the apical compartment of the Transwell inserts in the holding plate then remove it by aspiration.

4. Rinse inserts in the holding plate with $500 \mu \mathrm{L}$ of warmed DPBS using a P1000 pipette.

5. Add $500 \mu \mathrm{L}$ of warmed cell culture media to your sample inserts using a P1000 pipette.

6. Transfer your sample insert into the EndOhm-12 cup chamber.

a. Check for bubbles underneath the blank insert.

b. Remove the insert and transfer the insert back into the EndOhm-12 cup to remove any bubbles.

c. Placing the insert into the EndOhm-12 cup at an angle can reduce the frequency of bubbles. 
7. Plug the EVOM2 connecting wires into the EndOhm-12 cup chamber, place the EndOhm-12 into a cell culture incubator, and close the door (Figure 1).

8. Switch the EVOM2 on.

a. Resistance readings should stabilize within 5 minutes.

b. Resistance stabilization time is cell type and cell condition dependent.

9. Switch the EVOM2 off and return the EndOhm-12 to the BSC.

10. Exchange sample inserts and repeat steps 7-9.

11. Return the sample insert set from the Holding Plate to the original Sample Plate.

a. Repeat steps 2-9 with the remaining samples.

\section{Clean up}

1. Return the EVOM2 unit to its box.

2. Aspirate all media out of the EndOhm-12 cup chamber.

3. Rinse both the EndOhm-12 cup chamber and the EndOhm-12 cap probe with ddH2O, spray with $70 \%$ Ethanol, and rinse one final time with $\mathrm{ddH} 2 \mathrm{O}$.

a. Ensure no water floods the electronic connection ports.

4. Allow the EndOhm-12 cup chamber to air dry. 
5. Return the EndOhm-12 Unit to its bubble wrap and storage box.

\section{Data analysis}

1. TEER values are expressed as $\mathrm{W} \mathrm{cm}^{2}$, which can be determined by multiplying the resistance values obtained above (in W) by the surface area of the insert in $\mathrm{cm}^{2}$ (Corning $12 \mathrm{~mm}$ insert surface area $=1.12$ $\mathrm{cm}^{2}$ ).

2. Determine the mean TEER of the blank samples and subtract this value from all of the sample TEER values.

3. TEER data and differences/changes in TEER should be reported as actual TEER values (using the units of $\mathrm{W} \mathrm{cm}^{2}$ ) and not fold or percent changes.

\section{QUALITY CONTROL RATIONALE}

If the equipment is not cared for or calibrated it will result in incorrect readings. Use the provided test resistor to test the EVOM prior to each round of data collection. The test resistor is $1000 \mathrm{~W}$. If test resistor does not read $1000 \mathrm{~W}$ then use a screwdriver to adjust the EVOM unit in the R ADJ port.

\section{References}

EVOM2 Instruction Manual:

https://www.wpiinc.com/clientuploads/pdf/EVOM2_IM.pdf

\section{EndOhm-12 Instruction Manual:}

https://www.wpi-europe.com/downloads/content/EndOhm_IMs.pdf

\section{Figures}




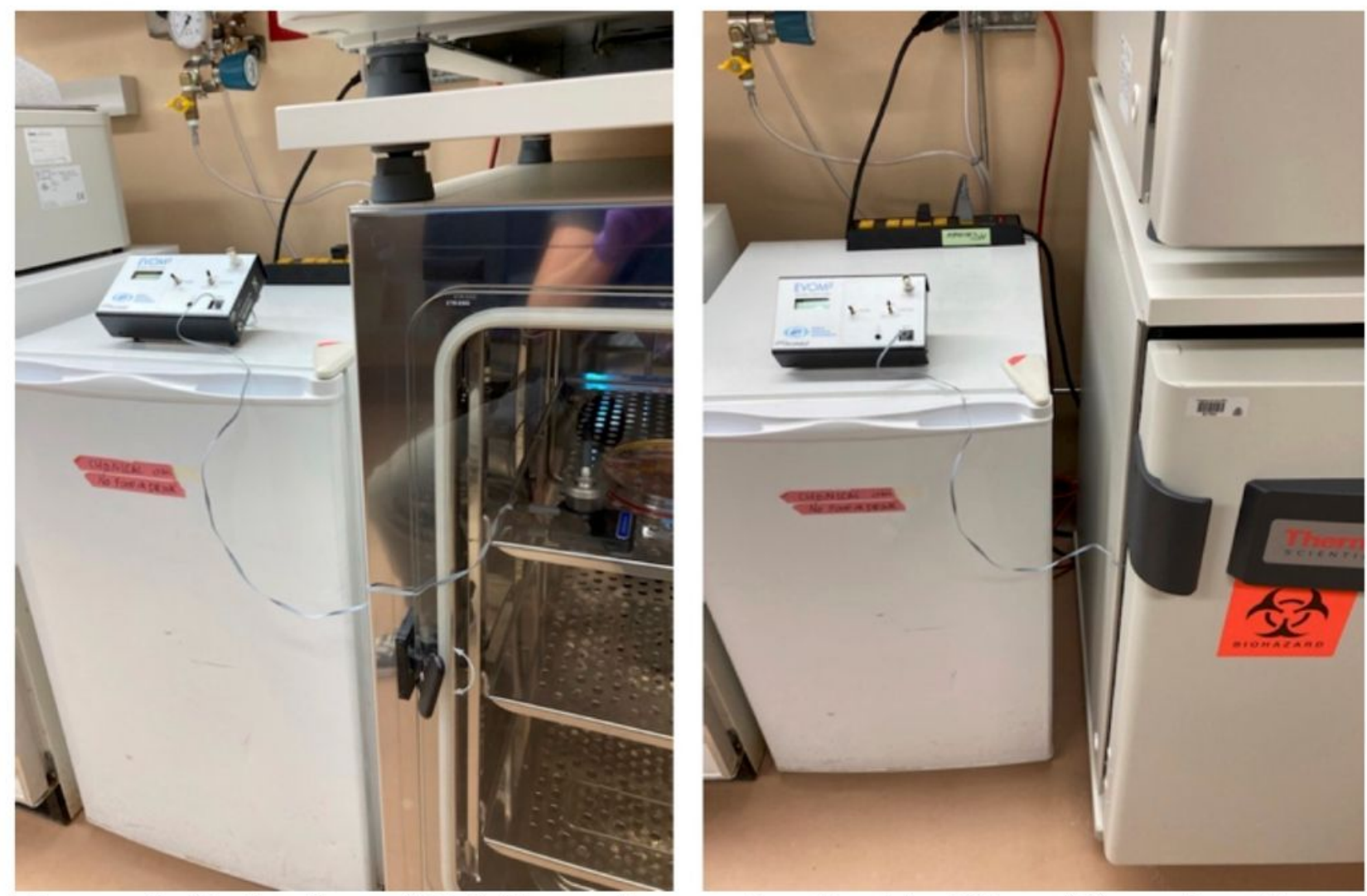

Figure 1. Collection of TEER readings using the EndOhm-12 cup in a tissue culture incubator

Figure 1

Collection of TEER readings using the EndOhm-12 cup in a tissue culture incubator

\section{Supplementary Files}

This is a list of supplementary files associated with this preprint. Click to download.

- 2020MeasurementofTransEpithelialElectricalResistancewithEndOhmChamberV1.pdf 